\title{
On the distribution of primitive abundant numbers
}

\author{
by
}

\author{
Michael R. Avidon (Atlanta, Ga.)
}

A number $m$ is primitive abundant if it is abundant $(\sigma(m) \geq 2 m)$, and all its proper divisors $d$ are deficient $(\sigma(d)<2 d)$, where $\sigma(m)$ is the sum of the divisors of $m$. Let $P(n)$ represent the number of primitive abundant numbers (p.a.n.) $\leq n$. In 1935, Erdős [2] proved the following result. For $n$ sufficiently large,

$$
n \cdot \exp \left[-c_{1} \sqrt{\log n \cdot \log \log n}\right] \leq P(n) \leq n \cdot \exp \left[-c_{2} \sqrt{\log n \cdot \log \log n}\right]
$$

with $c_{1}=8$ and $c_{2}=1 / 25$. In 1985, Ivić [4] improved this, proving the inequalities with $c_{1}=\sqrt{6}+\varepsilon$ and $c_{2}=1 / \sqrt{12}-\varepsilon$. In this paper, we improve it to the following.

$$
\begin{aligned}
& \text { Theorem. For } n \geq n_{0}(\varepsilon) \\
& \begin{aligned}
n \cdot \exp [-(\sqrt{2}+\varepsilon) \sqrt{\log n \cdot \log \log n}] & \leq P(n) \\
& \leq n \cdot \exp [-(1-\varepsilon) \sqrt{\log n \cdot \log \log n}] .
\end{aligned}
\end{aligned}
$$

I would like to thank Professor Carl Pomerance for suggesting the problem and for his abundant assistance.

The following notation will be standard throughout:

- $h(m)=\sigma(m) / m$,

- $E=e^{\sqrt{\log n \cdot \log \log n}}, L=\sqrt{\log n / \log \log n}$,

- $p_{1}=p_{1}(m)=$ largest prime divisor of $m$,

- $q=$ largest squarefull divisor of $m$

( $n$ is squarefull means $p\left|n \Rightarrow p^{2}\right| n$ for all primes $p$ ),

- $f=$ squarefree part of $m$; i.e. $f=m / q$,

- $p, p_{j}=$ prime numbers,

- $\varepsilon=$ arbitrarily small, positive numbers, not necessarily the same at each occurrence.

The method of proof, for both bounds, is a refinement of the one in [2] and [4]. For the upper bound, rather than divide into 3 cases (small $p_{1}$, 
large $q$, or large $p_{1}$ and small $q$ ), we divide into many cases, where both $p_{1}$ and $q$ are restricted to short intervals. We are consequently able to combine either 2 or 3 bounds from the 3 original cases (Lemmas 7 and 9). Further, in the main case (large $p_{1}$ and small $q$ ), we are able to get an improvement by removing the restriction that the map constructed is 1-1 (Lemmas 10 and 11).

As for the lower bound, we still consider only numbers of the form $2^{l} \cdot p_{k} \ldots p_{1}$. Rather than choosing all the primes from one small interval, the smallest is chosen to essentially be as small as possible, which has the effect of allowing the others to be chosen larger; hence there are more choices. At the same time, one must still restrict each to a short interval, to get sharp approximations of $h\left(p_{j}\right)$.

The upper bound. We first state some results from other papers that are used.

Lemma 1. Let $F(x)$ be the number of squarefull numbers $n \leq x$. Then

$$
F(x) \sim \frac{\zeta(3 / 2)}{\zeta(3)} x^{1 / 2}
$$

This result is proved in [3].

Lemma 2. Let $\psi(x, y)=\sum_{n \leq x, p_{1}(n) \leq y} 1$ and $u=\log x / \log y$. Suppose $\log x<y<x^{o(1)}$. Then

$$
\psi(x, y) \leq x / u^{(1+o(1)) u} .
$$

This result is proved in [1].

Lemma 3. Suppose $\eta \geq 1, m=p_{1} \ldots p_{t}, p_{1} \geq \ldots \geq p_{t}$ and

$$
p_{i} \leq \eta \cdot\left(p_{i+1} \ldots p_{t}\right), \quad 1 \leq i \leq t-1 .
$$

Then for any $D$ with $1 \leq D<m$, there exists $d \mid m$ with

$$
D /\left(\eta p_{t}\right)<d \leq D \text {. }
$$

This is equivalent to Lemma 4 of [5], with $a_{i}=\log p_{i}$.

We now prove some preliminary results.

Lemma 4. If $m$ is a p.a.n., $m \leq n$, and $m=p_{1} \ldots p_{t}$, where $p_{1} \geq \ldots$ $\geq p_{t}$, then

$$
p_{i} \leq 2 \log n \cdot p_{i+1} \ldots p_{t}+1, \quad i=1, \ldots, t .
$$

Proof. Let $v=p_{1} \ldots p_{i}$ and $u=p_{i+1} \ldots p_{t}$ (if $i=t$, then $u=1$ ). If the lemma is false, there exists $i$ such that $p_{i}-1>2 \log n \cdot u$. Note that $u$ 
is deficient, and since $v \leq n$, the number of distinct prime divisors $v$ has is $\leq \log n$. Thus

$$
\begin{aligned}
h(m) & =h(u) h(v) \leq\left(2-\frac{1}{u}\right) \prod_{p \mid v}\left(1+\frac{1}{p-1}\right) \\
& <\left(2-\frac{1}{u}\right) \prod_{p \mid v}\left(1+\frac{1}{2 \log n \cdot u}\right)<\left(2-\frac{1}{u}\right)\left(1+\frac{1}{2 \log n \cdot u}\right)^{\log n} \\
& <\left(2-\frac{1}{u}\right)\left(1+\frac{1}{2 u}+\frac{1}{(2 u)^{2}}+\ldots\right)=2 .
\end{aligned}
$$

This contradicts the abundance of $m$.

Corollary. If $m$ is a p.a.n., $m \leq n$, and $1 \leq D<m$, then there exists $d \mid m$ with

$$
D /(2 \log n+1)^{2}<d \leq D .
$$

Proof. This follows immediately from Lemmas 3 and 4 , since $p_{t} \leq$ $2 \log n+1$.

LEMma 5. Let $S$ be the set of $m$ that satisfy (i) $m \leq n$ and (ii) $q \geq E^{\zeta}$. Then

$$
|S| \ll n / E^{\zeta / 2} .
$$

Proof. Using Lemma 1 and partial summation we obtain

$$
\begin{aligned}
\sum_{\substack{m \leq n \\
q \geq E^{\zeta}}} 1 \leq \sum_{E^{\zeta} \leq q \leq n} \frac{n}{q}=\sum_{E^{\zeta} \leq q \leq n} 1+n \int_{E^{\zeta}}^{n}\left(\sum_{E^{\zeta} \leq q \leq t} 1\right) \frac{d t}{t^{2}} \\
\ll n^{1 / 2}+n \int_{E^{\zeta}}^{n} t^{-3 / 2} d t \ll n \cdot E^{-\zeta / 2} .
\end{aligned}
$$

LEMma 6. Let $S$ be the set of $m$ that satisfy (i) $m \leq n$ and (ii) $p_{1} \leq E^{\beta}$. Then for each $\varepsilon>0$ there is a number $n_{0}(\varepsilon)$ such that if $n \geq n_{0}(\varepsilon)$ then

$$
|S| \leq n / E^{1 /(2 \beta)-\varepsilon} .
$$

Pro of. This follows from Lemma 2 with $x=n, y=E^{\beta}$, since

$$
\begin{aligned}
u^{u} & =\exp [u \cdot \log u] \\
& \geq \exp \left[\frac{\log n}{\beta \sqrt{\log n \cdot \log \log n}} \log \left([\log n]^{1 / 2-\varepsilon}\right)\right] \\
& =\exp \left[\frac{1 / 2-\varepsilon}{\beta} \sqrt{\log n \cdot \log \log n}\right] .
\end{aligned}
$$


LEMma 7. Let $S$ be the set of $m$ that satisfy (i) $m \leq n$, (ii) $p_{1} \leq E^{\beta}$, and (iii) $E^{\zeta} \leq q$. Then for each $\varepsilon>0$ there is a number $n_{0}(\varepsilon)$ such that if $n \geq n_{0}(\varepsilon)$ then

$$
|S| \leq n / E^{1 /(2 \beta)+\zeta / 2-\varepsilon}
$$

Proof. By Lemma 5, we may assume that $q<E^{1 / \beta+\zeta}$. Then we may apply Lemma 6 to obtain

$$
\psi\left(n / q ; E^{\beta}\right) \leq \frac{n / q}{E^{1 /(2 \beta)-\varepsilon}} .
$$

Then, as in Lemma 5,

$$
\begin{aligned}
\sum_{\substack{m \leq n, p_{1} \leq E^{\beta} \\
E^{\zeta} \leq q<E^{1 / \beta+\zeta}}} 1 \leq \sum_{E^{\zeta} \leq q<E^{1 / \beta+\zeta}} \psi\left(n / q ; E^{\beta}\right) \\
\leq \frac{1}{E^{1 /(2 \beta)-\varepsilon}} \sum_{E^{\zeta} \leq q<E^{1 / \beta+\zeta}} \frac{n}{q} \leq \frac{n}{E^{1 /(2 \beta)+\zeta / 2-\varepsilon}} .
\end{aligned}
$$

LemmA 8. Let $m$ be a p.a.n. with $p_{1} \geq E^{\beta}$ and $p_{1}^{2} \nmid m$. Then

$$
2 \leq h(m)<2+2 / E^{\beta} .
$$

Proof. Since $p_{1}^{2} \nmid m$, we have $\left(m / p_{1}, p_{1}\right)=1$. Also $m / p_{1}$ is deficient. Therefore

$$
h(m)=h\left(m / p_{1}\right) h\left(p_{1}\right)<2\left(1+1 / p_{1}\right) \leq 2\left(1+1 / E^{\beta}\right) .
$$

Lemma 9. Let $S$ be the set of p.a.n.s $m$ that satisfy (i) $m \leq n$, (ii) $E^{\beta} \leq p_{1} \leq E^{\alpha}, 0<\beta \leq \alpha$, (iii) $E^{\zeta} \leq q \leq E^{\gamma}, 0<\zeta \leq \gamma$, and $\beta>\gamma / 2$, (iv) there exists $d \mid f$ such that $E^{c} \leq d \leq \frac{1}{2} E^{\beta / 2}$. Then for each $\varepsilon>0$ there is a number $n_{0}(\varepsilon)$ such that if $n \geq n_{0}(\varepsilon)$ then

$$
|S| \leq n / E^{c+1 /(2 \alpha)+\zeta / 2-\varepsilon} .
$$

Proof. Define a map from $S$ to $\left[1, n / E^{c}\right]$ by $m \mapsto m / d$. We claim that this map is $1-1$.

If $m_{1} \neq m_{2}$ and $d_{1}=d_{2}$ then $m_{1} / d_{1} \neq m_{2} / d_{2}$. So consider $m_{1} \neq m_{2}$ and $d_{1} \neq d_{2}$ and suppose that $m_{1} / d_{1}=m_{2} / d_{2}$. Then

$$
h\left(m_{1} / d_{1}\right)=h\left(m_{2} / d_{2}\right) .
$$

Since $\left(m_{i} / d_{i}, d_{i}\right)=1$ we have

$$
h\left(m_{i}\right)=h\left(m_{i} / d_{i}\right) h\left(d_{i}\right), \quad i=1,2 .
$$

It follows that

$$
\frac{h\left(d_{1}\right)}{h\left(d_{2}\right)}=\frac{h\left(m_{1}\right)}{h\left(m_{2}\right)}<\frac{2+2 / E^{\beta}}{2}=1+\frac{1}{E^{\beta}}
$$

using Lemma 8 (which is valid by (ii) and (iii)). 
On the other hand, since $d_{1}$ and $d_{2}$ are squarefree, $h\left(d_{1}\right) \neq h\left(d_{2}\right)$. Therefore, we may assume that $h\left(d_{1}\right) / h\left(d_{2}\right)>1$. Thus, since $d_{2}$ is deficient,

$$
\frac{h\left(d_{1}\right)}{h\left(d_{2}\right)}=\frac{\sigma\left(d_{1}\right) d_{2}}{d_{1} \sigma\left(d_{2}\right)} \geq 1+\frac{1}{d_{1} \sigma\left(d_{2}\right)}>1+\frac{1}{2 d_{1} d_{2}} \geq 1+\frac{2}{E^{\beta}}
$$

by (iv), which is a contradiction. Hence, the map is 1-1.

This gives us a 1-1 correspondence between $S$ and a subset $T$ of $\left[1, n / E^{c}\right]$. Since $d \mid f$, the squarefull part of $m / d$ is the same as that of $m$, and since $d \leq \frac{1}{2} E^{\beta / 2}, p_{1}$ is the same for both. Thus $m / d$ satisfies (ii) and (iii). Applying Lemma 7 to $T$ yields the result.

Lemma 10. Let $S$ be the set of p.a.n.s $m$ that satisfy (i) $m \leq n$, (ii) $E^{\beta} \leq p_{1} \leq E^{\alpha}$, (iii) $E^{\zeta} \leq q \leq E^{\gamma}$, where $\beta>\gamma / 2$, (iv) there exists $d \mid f$ such that $E^{\lambda} \leq d \leq E^{\eta}$, where $\eta \geq \beta / 2$. Then

$$
|S| \leq n / E^{2 \lambda-3 \eta+\beta+1 /(2 \alpha)+\zeta / 2-\varepsilon} .
$$

Proof. We follow the proof of Lemma 9, except that the map is not necessarily 1-1. Suppose it is at worst $N$ to 1 . If $d_{1}$ and $d_{2}$ are divisors as in (iv), we know that repeats satisfy

$$
1+\frac{1}{E^{\beta}}>\frac{\sigma\left(d_{1}\right) d_{2}}{d_{1} \sigma\left(d_{2}\right)}>1
$$

with $\sigma\left(d_{i}\right)<2 d_{i}$. It follows that

$$
\frac{2 d_{1} d_{2}}{E^{\beta}}>\sigma\left(d_{1}\right) d_{2}-d_{1} \sigma\left(d_{2}\right)>0
$$

and thus, by (iv),

$$
\frac{2 E^{2 \eta-\beta}}{\tau}>D>0
$$

where

$$
D=\frac{\sigma\left(d_{1}\right)}{\tau} d_{2}-\frac{d_{1}}{\tau} \sigma\left(d_{2}\right) \quad \text { and } \quad \tau=\left(d_{1}, \sigma\left(d_{1}\right)\right) .
$$

For given values of $D$ and $d_{1}, d_{2}$ is fixed $\bmod d_{1} / \tau$, so the number of possibilities for $d_{2}$ is

$$
<\frac{E^{\eta}}{d_{1} / \tau} \leq E^{\eta-\lambda} \tau
$$

Thus, given $d_{1}$, the total number of possibilities for $d_{2}$ is

$$
<\frac{2 E^{2 \eta-\beta}}{\tau} E^{\eta-\lambda} \tau=2 E^{3 \eta-\lambda-\beta}
$$

This is a bound for $N$. It follows, as in Lemma 9, that

$$
|S| \leq 2 E^{3 \eta-\lambda-\beta} \frac{n}{E^{\lambda+1 /(2 \alpha)+\zeta / 2-\varepsilon}},
$$

and the result follows. 
Lemma 11. Let $S$ be the set of p.a.n.s $m$ that satisfy (i) $m \leq n$, (ii) $E^{\beta} \leq p_{1} \leq E^{\beta+\varepsilon}$, (iii) $E^{\zeta} \leq q \leq E^{\zeta+\varepsilon}$, where $\beta>(\zeta+\varepsilon) / 2$, (iv) there exists $d \mid f$ with $E^{\beta / 2-\zeta / 4} \leq d \leq E^{\beta / 2+\zeta / 2-\delta}$, where $\delta=(\zeta / 2) \cdot(2 / 3)^{J-1}$ and $J=E^{o(1)}$. Then

$$
|S| \leq n / E^{1-\varepsilon} .
$$

Proof. Let $\eta_{j}=\beta / 2+\zeta / 2-(3 / 2)^{j-1} \delta$ and $\lambda_{j}=\eta_{j+1}$ for $j=1, \ldots, J$. Note that $\eta_{J}=\beta / 2$. Let $S_{j}$ be the set of p.a.n.s that satisfy (i)-(iii) and for which there exists $d \mid f$ with $E^{\lambda_{j}} \leq d \leq E^{\eta_{j}}$. Then $S=\bigcup_{j=1}^{J} S_{j}$ and therefore $|S| \leq \sum_{j=1}^{J}\left|S_{j}\right|$. Now Lemma 10 applies to $S_{j}$ and

$$
2 \lambda_{j}-3 \eta_{j}+\beta+1 /(2 \beta)+\zeta / 2-\varepsilon=\beta / 2+1 /(2 \beta)-\varepsilon
$$

so it follows that

$$
\left|S_{j}\right| \leq n / E^{\beta / 2+1 /(2 \beta)-\varepsilon} \leq n / E^{1-\varepsilon} .
$$

Since $J=E^{o(1)}$, the result follows.

LEMMA 12. Let $S$ be the set of p.a.n.s $m$ that satisfy (i) $m \leq n$, (ii) $E^{\beta} \leq p_{1} \leq E^{\beta+\varepsilon}$, (iii) $E^{\zeta} \leq q \leq E^{\zeta+\varepsilon}$, where $\beta>(\zeta+\varepsilon) / 2$, (iv) there exists $d \mid f$ with $E^{\beta / 2-\zeta / 2-\delta-\varepsilon} \leq d \leq E^{\beta / 2-\zeta / 4}$, where $\delta=o(1)$. Then

$$
|S| \leq n / E^{1-\varepsilon} .
$$

P r o of. This follows immediately from Lemma 9 , since $\min (\beta / 2+1 /(2 \beta))$ $=1$.

We now proceed to establish

$$
P(n) \leq n / E^{1-\varepsilon} .
$$

First, by Lemma 5 , those $m$ with $q \geq E^{2}$ can be ignored. Next, it follows from the Corollary to Lemma 4 that if $q<E^{2}$, there exists $d \mid f$ with $E^{1-\varepsilon}<d \leq \frac{1}{2} E^{3}$. Thus, by Lemma 9 , those $m$ with $p_{1} \geq E^{6}$ and $q<E^{2}$ also can be ignored.

We take the remaining set of p.a.n.s $m \leq n$, with $p_{1}<E^{6}$ and $q<E^{2}$, divide it into $\ll(\log n)^{2}=E^{o(1)}$ subsets, and establish the desired bound on each of these.

Specifically, let $S_{j, k}$ be the set of p.a.n.s $m \leq n$, with

$$
e^{j / L} \leq p_{1} \leq e^{(j+1) / L} \quad \text { and } \quad e^{k / L} \leq q \leq e^{(k+1) / L}
$$

for $0 \leq j \leq 6 \log n$ and $0 \leq k \leq 2 \log n$.

First, we will handle those $S_{j, k}$ with $j<k$. Noting that, with $\beta=$ $(j+1) / \log n$ and $\zeta=k / \log n$, we have $\beta-\varepsilon<\zeta$, Lemma 7 yields

$$
\left|S_{j, k}\right| \leq n / E^{1 /(2 \beta)+\beta / 2-\varepsilon} \leq n / E^{1-\varepsilon} .
$$

Now, for those with $j \geq k$, we will use Lemmas 11 and 12 . First note that, by the Corollary to Lemma 4 , if $q \leq E^{\zeta+\varepsilon}$ then there exists a $d \mid f$ 
with $E^{\beta / 2-\zeta / 2-\delta-\varepsilon} \leq d \leq E^{\beta / 2+\zeta / 2-\delta}$. With $J$ and $\delta$ as in Lemma 11, let $J=\log n=E^{o(1)}$, so that $\delta=o(1)$. Since $j \geq k$, setting $\beta=j / \log n$ and $\zeta=k / \log n$, we have $\beta \geq \zeta$. Thus the conditions of Lemmas 11 or 12 are satisfied by the p.a.n.s in $S_{j, k}$, and hence we have the desired bound.

The lower bound. Here we construct a set of numbers, show them to be primitive abundant, and underestimate the cardinality of the set. We first need to define many parameters.

$$
\alpha \in(\sqrt{2}-1 / L, \sqrt{2}]
$$

is chosen so that

$$
k=\alpha L \in \mathbb{N} .
$$

Now define

$$
\begin{gathered}
\delta=\frac{1}{8 k e^{k}}, \\
t=\left[8 k^{2} e^{k}\right] .
\end{gathered}
$$

We consider sequences $\left(k_{j}\right)$ with

$$
k_{j} \in\{0,1\}, \quad \sum_{j=1}^{t} k_{j}=k-2, \quad \text { and } \quad k_{t+1}=1 .
$$

For each sequence we will define a set of numbers which will be shown to be primitive abundant. These sets will be disjoint, so $P(n)$ is at least the sum of their cardinalities. Now define $\beta$ by

$$
n=8 E^{\beta(k+1)}(4 k)^{k-1}(1+\delta)^{\Sigma_{j=1}^{t+1} j k_{j}} .
$$

We can now define

$$
\begin{gathered}
l=\left[\frac{\beta \log E}{\log 2}\right], \\
S=\sum_{j=1}^{t} \frac{k_{j}}{2 k(1+\delta)^{j-1}} .
\end{gathered}
$$

Note that, by (5),

$$
0<S<1 / 2 .
$$

We claim that it follows from (6) that

$$
\beta=1 / \sqrt{2}+o(1)
$$

Therefore, from (2) and (7),

$$
k=o(l) .
$$


Indeed, since we have $n=E^{L}$ from the definitions of $E$ and $L,(6)$ implies

$$
E^{\beta k}<E^{L}<E^{\beta k+\beta} e^{k \log 4 k} e^{t k \delta}<E^{\beta k+\beta} e^{k^{2}(1+\varepsilon)}=E^{\beta k+o(k)} .
$$

Thus $\beta \alpha L<L<\beta \alpha L+o(L)$ by (2), which implies $1-o(1)<\beta \alpha<1$.

Our set of numbers will be those of the form $m=2^{l} p_{k} \ldots p_{1}$, where $p_{k}<p_{k-1}<\ldots<p_{1}$ are primes chosen as follows:

$$
p_{k} \in\left[\frac{1}{1-S} 2^{l+1}, \frac{1+\delta}{1-S} 2^{l+1}\right] .
$$

We define intervals $I_{j}$ by

$$
I_{j}=\left[k(1+\delta)^{j-1} 2^{l+2}, k(1+\delta)^{j} 2^{l+2}\right), \quad j=1, \ldots, t,
$$

and we choose $k_{j}$ primes from $I_{j}$ to give us $p_{k-1}, \ldots, p_{2}$. Finally, we choose $p_{1}$ with

$$
p_{1} \in\left[k(1+\delta)^{t} 2^{l+2}, k(1+\delta)^{t} 2^{l+3}\right] .
$$

Note that, for any number $m$ with $p_{1} \geq 2 q$, which holds for the numbers $m$ of the above form (here $q=2^{l}$ ), if we have $h\left(m / p_{1}\right)<2$ then it follows that $h(d)<2$ for all proper divisors $d$ of $m$. Thus, we need to establish:
(A) $h\left(m / p_{1}\right)<2$,
(B) $h(m) \geq 2$,
(C) $m \leq n$,
(D) $\#\{m\} \geq n / E^{\sqrt{2}+\varepsilon}$.

Proof of (A). Since $q=2^{l}$,

$$
h(q)=2-\frac{1}{2^{l}}=2\left(1-\frac{1}{2^{l+1}}\right) .
$$

From (12) we have

$$
h\left(p_{k}\right)=1+\frac{1}{p_{k}} \leq 1+\frac{1-S}{2^{l+1}} .
$$

From (13) we have

$$
h\left(p_{k-1} \ldots p_{2}\right) \leq \prod_{j=1}^{t}\left(1+\frac{k_{j}}{k(1+\delta)^{j-1} \cdot 2^{l+2}}\right) .
$$

Note that $\prod\left(1+\varepsilon_{i}\right)<1+\sum \varepsilon_{i}+\left(\sum \varepsilon_{i}\right)^{2}$ when each $\varepsilon_{i}>0$ and $\sum \varepsilon_{i}<1 / 2$. Using this fact with (8) and the above, we have

$$
h\left(p_{k-1} \ldots p_{2}\right)<1+\frac{S}{2^{l+1}}+\frac{S^{2}}{2^{2 l+2}} .
$$


Combining (15)-(17) with (9), we have

$$
\begin{aligned}
h\left(m / p_{1}\right) & =h(q) h\left(p_{k}\right) h\left(p_{k-1} \ldots p_{2}\right) \\
& \leq 2\left(1-\frac{1}{2^{l+1}}\right)\left(1+\frac{1-S}{2^{l+1}}\right)\left(1+\frac{S}{2^{l+1}}+\frac{S^{2}}{2^{2 l+2}}\right) \\
& =2\left(1-\frac{1}{2^{l+1}}\right)\left[1+\frac{1}{2^{l+1}}+\frac{S}{2^{2 l+2}}+O\left(\frac{1}{2^{3 l}}\right)\right] \\
& <2\left[1-\frac{1}{2^{2 l+2}}+\frac{1}{2^{2 l+3}}+O\left(\frac{1}{2^{3 l}}\right)\right]<2 .
\end{aligned}
$$

Proof of (B). From (12) and (14) we have

$$
\begin{aligned}
& h\left(p_{k}\right) \geq 1+\frac{1-S}{(1+\delta) \cdot 2^{l+1}}, \\
& h\left(p_{1}\right) \geq 1+\frac{1}{k(1+\delta)^{t} \cdot 2^{l+3}} .
\end{aligned}
$$

From (13) and (8) we have

$$
\begin{aligned}
h\left(p_{k-1} \ldots p_{2}\right) & >\prod_{j=1}^{t}\left(1+\frac{k_{j}}{k(1+\delta)^{j} \cdot 2^{l+2}}\right) \\
& >1+\sum_{j=1}^{t} \frac{k_{j}}{2 k(1+\delta)^{j} \cdot 2^{l+1}}=1+\frac{S}{(1+\delta) \cdot 2^{l+1}} .
\end{aligned}
$$

Combining (15), (18)-(20) and (9) gives

$$
\begin{aligned}
h(m)> & 2\left(1-\frac{1}{2^{l+1}}\right)\left(1+\frac{1-S}{(1+\delta) \cdot 2^{l+1}}\right)\left(1+\frac{S}{(1+\delta) \cdot 2^{l+1}}\right) \\
& \times\left(1+\frac{1}{k(1+\delta)^{t} \cdot 2^{l+3}}\right) \\
= & 2\left[1+\left(-1+\frac{1-S}{1+\delta}+\frac{S}{1+\delta}+\frac{1}{4 k(1+\delta)^{t}}\right) \frac{1}{2^{l+1}}+O\left(\frac{1}{2^{2 l}}\right)\right] \\
= & 2\left[1+\left(\frac{-\delta}{1+\delta}+\frac{1}{4 k(1+\delta)^{t}}\right) \frac{1}{2^{l+1}}+O\left(\frac{1}{2^{2 l}}\right)\right] .
\end{aligned}
$$

By (3) and (4), $(1+\delta)^{t}<(1+\delta)^{k / \delta}<e^{k}$ and therefore

$$
\frac{1}{4 k(1+\delta)^{t}}-\frac{\delta}{1+\delta}>\frac{1}{4 k e^{k}}-\frac{1}{8 k e^{k}}=\frac{1}{8 k e^{k}} .
$$

It follows from $(11)$ that $h(m)>2$. 
Proof of (C). Using (7), (12)-(14), and (9) we have

$$
\begin{aligned}
m & =2^{l} p_{k}\left(p_{k-1} \ldots p_{2}\right) p_{1} \\
& \leq E^{\beta} \cdot 4(1+\delta) E^{\beta}\left(4 k E^{\beta}\right)^{k-2}(1+\delta)^{\Sigma_{j=1}^{t} j k_{j}} \cdot 8 k E^{\beta}(1+\delta)^{t} \\
& =8 E^{\beta(k+1)}(4 k)^{k-1}(1+\delta)^{\Sigma_{j=1}^{t+1} j k_{j}} .
\end{aligned}
$$

Thus, by $(6), m \leq n$.

Proof of (D). By (12)-(14), (7) and the prime number theorem,

$$
P(n) \gg \sum_{\left(k_{j}\right)} \frac{\delta E^{\beta}}{\log E} \prod_{j=1}^{t}\left[\frac{4 \delta k(1+\delta)^{j-1} E^{\beta}}{\log \left(4 k(1+\delta)^{j} E^{\beta}\right)}\right]^{k_{j}} \frac{k(1+\delta)^{t} E^{\beta}}{\log \left(8 k(1+\delta)^{t} E^{\beta}\right)} .
$$

Note that (3), (4), (11), and (7) tell us

$$
4 k(1+\delta)^{j}<4 k(1+\delta)^{k / \delta}<4 k e^{k}=e^{o(l)}<E^{\beta} .
$$

Likewise, for any constant $c, c^{k}=E^{o(1)}$. Thus, using (5),

$$
P(n) \gg \sum_{\left(k_{j}\right)} \frac{\delta^{k-1} \cdot k^{k-1} \cdot E^{\beta k}(1+\delta)^{\Sigma_{j=1}^{t+1} j k_{j}}}{E^{o(1)} \cdot(\log E)^{k}} .
$$

Note that, by (2), $(\log E)^{k}=e^{\alpha L \log \log E}=e^{(\alpha / 2) L \log \log n(1+o(1))}=$ $E^{\alpha / 2+o(1)}$. Therefore, using (6) we find

$$
P(n) \gg \sum_{\left(k_{j}\right)} \frac{\delta^{k-1} \cdot n}{E^{\alpha / 2+\beta+o(1)}} .
$$

Applying (1), (10), (5), (3), and (4) shows that

$$
\begin{aligned}
P(n) & \gg \sum_{\left(k_{j}\right)} \frac{\delta^{k-1} n}{E^{\sqrt{2}+o(1)}}=\left(\begin{array}{c}
t \\
k-2
\end{array}\right) \delta^{k-1} \frac{n}{E^{\sqrt{2}+o(1)}} \\
& \geq\left(\frac{t}{k}\right)^{k-2} \delta^{k-1} \frac{n}{E^{\sqrt{2}+o(1)}}=\frac{n}{E^{\sqrt{2}+o(1)}} .
\end{aligned}
$$

\section{References}

[1] N. G. de Bruijn, On the number of integers $\leq x$ and free of prime factors $>y$, Indag. Math. 13 (1951), 50-60.

[2] P. Erdős, On primitive abundant numbers, J. London Math. Soc. 37 (1935), 49-58.

[3] P. Erdős und G. Szekeres, Über die Anzahl der abelschen Gruppen gegebener Ordnung und über ein verwandtes zahlentheoretisches Problem, Acta Sci. Math. (Szeged) 7 (1934), 95-102.

[4] A. Ivić, The distribution of primitive abundant numbers, Studia Sci. Math. Hungar. 20 (1985), 183-187. 
[5] C. Pomerance, On composite $n$ for which $\phi(n) \mid n-1, I I$, Pacific J. Math. 69 (1977), 177-186.

275.12 Street, Apt. 11

Atlanta, Georgia 30309

U.S.A.

Received on 10.8.1995

and in revised form on 17.10.1995 